OPEN ACCESS

Edited by:

Yves Poumay,

University of Namur, Belgium

Reviewed by: José Ascención Martínez-Álvarez, University of Guanajuato, Mexico

Cindy Staerck,

University of Liège, Belgium

*Correspondence:

Lin Ma

bch_maleen@aliyun.com

tThese authors have contributed equally to this work

Specialty section:

This article was submitted to Dermatology,

a section of the journa

Frontiers in Medicine

Received: 25 September 2021 Accepted: 08 November 2021 Published: 26 November 2021

Citation:

Zhou YB, Xiao YY, Chao JJ and Ma L (2021) In vitro Activity of Allicin Alone and in Combination With Antifungal Drugs Against Microsporum canis Isolated From Patients With Tinea

Capitis. Front. Med. 8:783086. doi: 10.3389/fmed.2021.783086

\section{In vitro Activity of Allicin Alone and in Combination With Antifungal Drugs Against Microsporum canis Isolated From Patients With Tinea Capitis}

\author{
Ya Bin Zhou ${ }^{\dagger}$, Yuan Yuan $\mathrm{Xiao}^{+}$, Jin Jing Chao and Lin Ma* \\ Department of Dermatology, Beijing Children's Hospital, Capital Medical University, National Center for Children's Health, \\ Beijing, China
}

The checkerboard broth method based on the Clinical and Laboratory Standards Institute M38-A3 document was used in this study to evaluate the in vitro activity of allicin alone and in combination with the antifungal drugs (griseofulvin, fluconazole, itraconazole and terbinafine) against Microsporum canis isolated from patients with tinea capitis. When allicin was used alone, only weak anti-M. canis effects were found. The $\mathrm{MIC}_{50}, \mathrm{MIC}_{90}$ and geometric mean (GM) of terbinafine were the lowest among the compounds tested. Synergism was observed for the combinations of allicin with itraconazole and terbinafine. Only indifference was observed for the combinations of allicin with griseofulvin and fluconazole. Our study illustrated the synergism of allicin in combination with itraconazole and terbinafine, which could be a reference for the treatment of tinea capitis due to M. canis.

Keywords: Microsporum canis, tinea capitis, allicin, drug combination, antifungal drug

\section{INTRODUCTION}

Tinea capitis is a common dermatophytosis of the scalp and hair follicles. A previous study on the epidemiology of tinea capitis in children suggested that Microsporum canis is the most prevalent pathogen in most areas of China (1).

Griseofulvin, terbinafine, fluconazole and itraconazole are most common used antifungal drugs for tinea capitis. However, resistance to antifungal drugs is emerging in M. canis (2). A strain of griseofulvin resistant $M$. canis with a high minimum inhibitory concentration (MIC) was isolated from recalcitrant tinea capitis which had treatment with griseofulvin (3). Recently, a strain of terbinafine resistant $M$. canis was isolated from a cat which failed to topical terbinafine treatment (4). High in vitro MICs of azoles were also been noted in M. canis (5). To cope with drug-resistant mycoses, a combination of traditional antifungal drugs with non-antifungal agents has been proposed as a promising treatment strategy (6).

Allicin, a sulfur compound from garlic, has been demonstrated to have activity against Candida, Cryptococcus, Trichophyton, Epidermophyton, and Microsporum (7). Although allicin and fluconazole are synergistic in vitro against Candida albicans (8), no studies have been conducted on the in vitro activity of allicin in combination with antifungal drugs against $M$. canis.

In the present study, the in vitro antifungal activity of allicin alone or in combination were assessed against 30 clinical $M$. canis strains. The results will help us evaluate the therapeutic potential of allicin in combination with antifungal drugs against tinea capitis. 


\section{MATERIALS AND METHODS}

\section{Microorganisms}

A total of $30 \mathrm{M}$. canis strains were tested. They were all recovered from the hair of patients with tinea capitis at the Outpatient Clinic of Dermatology (Beijing Children's Hospital, Capital Medical University). Each isolate was stored in 10\% glycerol at $-80^{\circ}$ C. Candida parapsilosis (ATCC 22019) and Trichophyton interdigitale (ATCC MYA 4439) were chosen as quality controls.

All strains were identified microscopically and with molecular sequencing of the internal transcribed spacer (ITS) region. Genomic DNA of each strain was extracted by using a Biospin Fungus Genomic DNA Extraction kit (Bioer Technology Ltd; Hubei, China) in accordance with the manufacturer's instructions. The extracted DNA was eluted with $100 \mu \mathrm{l}$ of distilled water, and $1 \mu \mathrm{l}$ of the extracted DNA was used for polymerase chain reaction (PCR). The ITS region was amplified using the primers ITS1 (5'-TCCGTAGGTGAACCTGCGG-3') and ITS4 (5'-GGTCCGTGTTTCAAGACGG-3'). Each PCR mixture contained $1 \mu \mathrm{l}$ of extracted fungal DNA, $0.08 \mu \mathrm{M}$ each of the primers, and $12.5 \mu \mathrm{l}$ of $2 \times$ Taq PCR MasterMix (Tiangen Biotech Ltd; Beijing, China) in $25 \mu \mathrm{l}$ of reaction volume. The PCR cycling comprised an initial denaturation at $95^{\circ} \mathrm{C}$ for $5 \mathrm{~min}$, followed by 35 cycles of $95^{\circ} \mathrm{C}$ for $30 \mathrm{~s}, 58^{\circ} \mathrm{C}$ for $30 \mathrm{~s}$, and $72^{\circ} \mathrm{C}$ for $1 \mathrm{~min}$, followed by a final extension at $72^{\circ} \mathrm{C}$ for $10 \mathrm{~min}$ and cooling to $4^{\circ} \mathrm{C}$. The amplicon products were then sequenced by Tian Yi Hui Yuan Company (Beijing, China). The sequences obtained were compared with those in the GenBank DNA database (https://blast.ncbi.nlm.nih.gov/). The sequences obtained had been deposited in GenBank with the following accession numbers: MT163398-MT163427.

\section{Chemicals}

All drugs were acquired from Harvey Biotech Ltd., (Beijing, China) and dissolved in dimethyl sulfoxide (DMSO). The stock solution concentrations were as follows: $12.8 \mathrm{mg} / \mathrm{mL}$ for allicin; $6.4 \mathrm{mg} / \mathrm{mL}$ for fluconazole; $1.6 \mathrm{mg} / \mathrm{mL}$ for itraconazole, griseofulvin and terbinafine. The drugs were analyzed in the following final concentration ranges: $2-128 \mu \mathrm{g} / \mathrm{mL}$ for allicin; $0.015-8 \mu \mathrm{g} / \mathrm{mL}$ for itraconazole and griseofulvin; $0.125-$ $64 \mu \mathrm{g} / \mathrm{mL}$ for fluconazole; and $0.001-0.5 \mu \mathrm{g} / \mathrm{mL}$ for terbinafine. The final concentration of DMSO did not exceed $1 \%$ in test wells.

\section{Checkerboard Microdilution Assay}

Assays were performed according to the checkerboard broth microdilution method based on the Clinical and Laboratory Standards Institute M38-A3 document $(9,10)$. Briefly, all strains enrolled in our study grew for 14 days on potato dextrose agar at $28^{\circ} \mathrm{C}$, and conidial suspensions were prepared by gently scraping the surfaces of the fungal colonies into sterile physiological saline. Heavy hyphae fragments were settled for $5 \mathrm{~min}$ at room temperature and the upper, conidial suspensions were used as an inoculum. Susceptibility tests were then performed in RPMI 1,640 medium (Gibco, USA) supplemented with $0.3 \mathrm{~g} / \mathrm{l} \mathrm{L-}$ glutamine but without sodium bicarbonate and buffered to $\mathrm{pH}$ 7.0 with $0.165 \mathrm{M}$ 4-morpholinopropanesulfonic acid (Amresco, USA). The final concentration of the suspension diffused in the wells was adjusted to $\sim 1-3 \times 10^{3} \mathrm{CFU} / \mathrm{mL}$, as determined with a hemocytometer. Serial 2-fold dilutions of $50 \mu \mathrm{l}$ of each drugs A (allicin) and B (fluconazole, itraconazole, griseofulvin or terbinafine) were dispensed along the vertical and horizontal directions to yield $100 \mu \mathrm{l}$ per well in a 96-well microtiter plate. One hundred microliters of the diluted inoculum suspension was dispensed into each well. The plates were incubated at $35^{\circ} \mathrm{C}$ for 4-5 days. The MICs were determined as the lowest concentration producing a $100 \%$ reduction in turbidity by visual observation with a concave mirror when compared with the drug-free control. All experiments were conducted in replicate on different days.

\section{Drug Interaction Analysis}

The drug combination interaction was evaluated on the basis of the fractional inhibitory concentration index (FICI), which is the sum of the MIC of each drug in combination divided by the MIC of the drug used alone. The drug interaction was defined as follows: FICI $\leq 0.5$, synergism; FICI $>0.5$ and $\leq 4.0$, indifference; and FICI $>4.0$, antagonism (9).

\section{RESULTS}

The results of the in vitro susceptibility tests of $M$. canis strains to the antifungal drugs alone are listed in Tables $\mathbf{1}, \mathbf{2}$. When allicin was used alone, only weak anti- $M$. canis effects were found (MICs, 16-128; GM, $46.313 \mu \mathrm{g} / \mathrm{ml}$ ). We also found that the $\mathrm{MIC}_{50}, \mathrm{MIC}_{90}$ and geometric mean (GM) of terbinafine were the lowest. Among the tested azoles, fluconazole had a higher $\mathrm{MIC}_{50}$, $\mathrm{MIC}_{90}$ and GM than itraconazole.

The results for each drug combination are listed in Tables 1, 3 . Synergism was observed in the following combinations: allicin + itraconazole $(86.7 \%)$ and allicin + terbinafine (80\%) (Tables $\mathbf{1}$, 3). When synergism was observed, the median reduction in itraconazole was 8 -fold (range 4 - to 32 -fold), while the median reduction in terbinafine was 16 -fold (range 4 - to 32-fold) (Table 1). Only indifference was observed in the following combinations: allicin + griseofulvin and allicin + fluconazole (Tables 1, 3).

\section{DISCUSSION}

Although many synthetic antifungal drugs are available for dermatophytosis, the occurrence of resistance or toxic sideeffects may lead to the treatment failure. Thus, new therapeutic strategies are necessary. Improvements in the efficacy of synthetic antifungal drugs and reductions in toxicity may be achieved using combination therapy with natural antifungal drugs. Previously studies have demonstrated the effects of herbal essential oils and their synergism with ketoconazole against Trichophyton spp (11). In this study, we investigated the in vitro antifungal activity of allicin alone and in combination with four antifungal agents (griseofulvin, fluconazole, itraconazole and terbinafine) against clinical isolates of $M$. canis.

Griseofulvin is the first systemic antifungal that was introduced for dermatophytosis. However, its use has been superseded by itraconazole and terbinafine in other types of 


\begin{tabular}{|c|c|c|c|c|c|c|c|c|c|c|c|c|c|}
\hline \multirow[t]{2}{*}{ Strains } & \multicolumn{3}{|c|}{ MIC $(\mu \mathrm{g} / \mathrm{mL})$} & \multirow[t]{2}{*}{ FICI } & \multicolumn{2}{|c|}{ MIC $(\mu \mathrm{g} / \mathrm{mL})$} & \multirow[t]{2}{*}{ FICI } & \multicolumn{2}{|c|}{ MIC $(\mu \mathrm{g} / \mathrm{mL})$} & \multirow[t]{2}{*}{ FICI } & \multicolumn{2}{|c|}{ MIC $(\mu \mathrm{g} / \mathrm{mL})$} & \multirow[t]{2}{*}{ FICI } \\
\hline & Allicin & Griseofulvin & Allicin/griseofulvin & & Fluconazole & Allicin/fluconazole & & Itraconazole & Allicin/itraconazole & & Terbinafine & Allicin/terbinafine & \\
\hline $\mathrm{BCH} 32585$ & 32 & 1 & $32 / 1$ & 2 & 16 & $32 / 16$ & 2 & 1 & $16 / 0.25$ & 0.75 & 0.06 & $8 / 0.004$ & 0.313 \\
\hline BCH32598 & 32 & 0.5 & $32 / 0.5$ & 2 & 32 & $16 / 16$ & 1 & 0.5 & $4 / 0.125$ & 0.375 & 0.015 & $4 / 0.002$ & 0.125 \\
\hline $\mathrm{BCH} 32773$ & 32 & 1 & $32 / 1$ & 2 & 64 & $32 / 64$ & 2 & 1 & $4 / 0.06$ & 0.188 & 0.015 & $8 / 0.004$ & 0.5 \\
\hline BCH32775 & 128 & 1 & $64 / 0.5$ & 1 & 32 & $32 / 16$ & 0.75 & 1 & $32 / 0.06$ & 0.313 & 0.03 & $16 / 0.004$ & 0.25 \\
\hline ВCH32785 & 64 & 0.5 & $32 / 0.25$ & 1 & 16 & $64 / 16$ & 2 & 0.5 & $16 / 0.03$ & 0.313 & 0.03 & 8/0.002 & 0.188 \\
\hline ВCH32820 & 64 & 0.5 & $64 / 0.5$ & 2 & 16 & $64 / 16$ & 2 & 1 & $4 / 0.25$ & 0.313 & 0.06 & 4/0.002 & 0.099 \\
\hline BCH32823 & 64 & 1 & $64 / 1$ & 2 & 64 & $32 / 8$ & 0.625 & 0.25 & $8 / 0.015$ & 0.188 & 0.008 & 16/0.002 & 0.5 \\
\hline BCH32860 & 32 & 0.25 & $32 / 0.25$ & 2 & 16 & $32 / 16$ & 2 & 0.25 & $4 / 0.015$ & 0.188 & 0.06 & $4 / 0.004$ & 0.188 \\
\hline BCH32931 & 32 & 1 & $16 / 0.5$ & 1 & 32 & $32 / 32$ & 2 & 0.25 & 8/0.06 & 0.5 & 0.004 & $16 / 0.001$ & 0.75 \\
\hline BCH33015 & 64 & 1 & $64 / 1$ & 2 & 16 & $64 / 16$ & 2 & 1 & $8 / 0.125$ & 0.25 & 0.06 & 8/0.002 & 0.156 \\
\hline ВCH33016 & 128 & 0.25 & $64 / 0.125$ & 1 & 16 & $64 / 8$ & 1 & 0.25 & $16 / 0.06$ & 0.375 & 0.015 & $16 / 0.001$ & 0.188 \\
\hline ВCH33022 & 64 & 0.25 & $32 / 0.125$ & 1 & 16 & $32 / 8$ & 1 & 0.25 & $8 / 0.06$ & 0.375 & 0.015 & 8/0.002 & 0.188 \\
\hline ВCH33034 & 32 & 1 & $32 / 1$ & 2 & 32 & $64 / 16$ & 2 & 1 & $8 / 0.125$ & 0.375 & 0.015 & $8 / 0.004$ & 0.5 \\
\hline ВCH33039 & 32 & 0.5 & $32 / 0.5$ & 2 & 16 & $32 / 16$ & 2 & 1 & $16 / 0.125$ & 0.75 & 0.03 & 4/0.002 & 0.188 \\
\hline BCH33042 & 64 & 0.5 & $32 / 0.125$ & 0.75 & 16 & $32 / 8$ & 1 & 1 & 8/0.03 & 0.156 & 0.008 & 16/0.001 & 0.375 \\
\hline ВСН33056 & 64 & 0.5 & 32/0.25 & 1 & 32 & $64 / 32$ & 2 & 0.5 & $16 / 0.03$ & 0.313 & 0.06 & $32 / 0.015$ & 0.75 \\
\hline ВСН33060 & 64 & 0.5 & $64 / 0.5$ & 2 & 16 & $32 / 8$ & 0.75 & 0.5 & $8 / 0.125$ & 0.375 & 0.015 & 4/0.002 & 0.188 \\
\hline BCH33061 & 32 & 0.5 & $16 / 0.125$ & 0.75 & 16 & $8 / 8$ & 0.75 & 1 & $8 / 0.125$ & 0.375 & 0.06 & 4/0.002 & 0.156 \\
\hline BCH33065 & 128 & 0.5 & $64 / 0.25$ & 1 & 16 & $64 / 8$ & 1 & 0.5 & $32 / 0.06$ & 0.375 & 0.03 & 16/0.002 & 0.188 \\
\hline BCH33072 & 64 & 0.5 & $64 / 0.5$ & 2 & 16 & $64 / 16$ & 2 & 1 & $16 / 0.125$ & 0.375 & 0.06 & $4 / 0.004$ & 0.125 \\
\hline BCH33073 & 32 & 1 & $32 / 1$ & 2 & 32 & $32 / 32$ & 2 & 0.5 & 8/0.06 & 0.5 & 0.03 & 8/0.008 & 0.5 \\
\hline BCH33082 & 16 & 0.5 & $16 / 0.5$ & 2 & 64 & $8 / 32$ & 1 & 0.25 & $2 / 0.03$ & 0.25 & 0.015 & 2/0.002 & 0.25 \\
\hline BCH33100 & 32 & 0.25 & $16 / 0.125$ & 1 & 16 & $16 / 8$ & 1 & 1 & $8 / 0.125$ & 0.375 & 0.06 & 4/0.002 & 0.156 \\
\hline BCH33105 & 16 & 1 & $16 / 1$ & 2 & 16 & $16 / 16$ & 2 & 0.5 & $4 / 0.06$ & 0.375 & 0.03 & $8 / 0.015$ & 1 \\
\hline ВCH33106 & 128 & 1 & $64 / 0.5$ & 1 & 32 & $128 / 32$ & 2 & 1 & $32 / 0.06$ & 0.313 & 0.06 & 16/0.002 & 0.156 \\
\hline $\mathrm{BCH} 33115$ & 64 & 0.25 & $64 / 0.25$ & 2 & 16 & $64 / 16$ & 2 & 0.5 & $16 / 0.03$ & 0.313 & 0.03 & 16/0.004 & 0.375 \\
\hline ВСН33122 & 16 & 1 & $8 / 0.125$ & 0.625 & 16 & $16 / 16$ & 2 & 1 & 2/0.125 & 0.25 & 0.06 & 8/0.03 & 1 \\
\hline BCH33126 & 32 & 0.5 & $32 / 0.5$ & 2 & 16 & $16 / 2$ & 0.625 & 1 & $16 / 0.125$ & 0.625 & 0.06 & 16/0.008 & 0.675 \\
\hline BCH33128 & 32 & 1 & $32 / 1$ & 2 & 64 & $32 / 64$ & 2 & 1 & $16 / 0.06$ & 0.563 & 0.008 & 16/0.002 & 0.75 \\
\hline $\mathrm{BCH} 33143$ & 64 & 1 & $32 / 0.25$ & 0.75 & 16 & $64 / 16$ & 2 & 0.5 & $8 / 0.015$ & 0.156 & 0.015 & 16/0.002 & 0.375 \\
\hline
\end{tabular}


TABLE 2 | MICs of allicin and antifungal drugs tested alone against $M$. canis strains $(\mu \mathrm{g} / \mathrm{mL})$.

\begin{tabular}{lcccc}
\hline Drug & MIC range & MIC $_{\mathbf{5 0}}$ & MIC $_{\mathbf{9 0}}$ & GM \\
\hline Allicin & $16-128$ & 32 & 128 & 46.313 \\
Griseofulvin & $0.25-1$ & 0.5 & 1 & 0.602 \\
Fluconazole & $16-64$ & 16 & 64 & 22.627 \\
Itraconazole & $0.25-1$ & 0.5 & 1 & 0.616 \\
Terbinafine & $0.004-0.06$ & 0.03 & 0.06 & 0.026 \\
\hline
\end{tabular}

TABLE 3 | MIC ranges of drug combinations, FICl ranges and synergistic ratios of tested $M$. canis strains $(\mu \mathrm{g} / \mathrm{mL})$.

\begin{tabular}{lccc}
\hline Antifungal combination & MIC range & FICl range & Synergistic ratio \\
\hline Allicin + griseofulvin & $8-64(0.125-1)$ & $0.625-2$ & 0 \\
Allicin + fluconazole & $8-128(2-64)$ & $0.625-2$ & 0 \\
Allicin + itraconazole & $2-32(0.015-0.25)$ & $0.156-0.75$ & $86.7 \%$ \\
Allicin + terbinafine & $2-32(0.001-0.06)$ & $0.099-1$ & $80 \%$ \\
\hline
\end{tabular}

dermatophytosis (12). Griseofulvin works by disrupting the mitotic spindle structure and inhibiting nucleic acid synthesis (13). Although griseofulvin-resistant M. canis strains has been documented in different studies $(3,5)$, no griseofulvin-resistant strains were detected in our study. In this study, the $\mathrm{MIC}_{50}$ and $\mathrm{MIC}_{90}$ of griseofulvin were 0.5 and $1 \mu \mathrm{g} / \mathrm{mL}$, which were consistent with a previous study from Iran (5). Although antagonism was not observed for the combination of allicin and griseofulvin, synergism was not observed either. Their combination in the clinic may not be suitable.

Azoles act on ergosterol biosynthesis by inhibiting $14 \alpha-$ demethylation of lanosterol, leading to altered permeability of the fungal membrane and defective fungal cell wall synthesis (2). In our study, fluconazole had a higher $\mathrm{MIC}_{50}, \mathrm{MIC}_{90}$ and GM than itraconazole, indicating that the latter might be superior for the treatment of tinea capitis. This result was consistent with a previous study from Iran (5). In vitro synergistic effects of allicin and fluconazole have been observed against $C$. albicans (8). Interestingly, this combination is indifferent against $M$. canis, which may be due to species differences. In addition, a previous study from Malaysia also demonstrated that allicin in combination with fluconazole showed indifferent interaction against $M$. canis (14). Allicin indeed exhibited synergistic effects in combination with itraconazole against $M$. canis (86.7\%).

Terbinafine inhibits the enzyme squalene epoxidase, blocking the synthesis of 2,3-oxidosqualene and thus leading to the accumulation of squalene and the depletion of ergosterol (2). Terbinafine had the lowest $\mathrm{MIC}_{50}(0.03 \mu \mathrm{g} / \mathrm{mL}), \mathrm{MIC}_{90}$ $(0.06 \mu \mathrm{g} / \mathrm{mL})$ and $\mathrm{GM}(0.026 \mu \mathrm{g} / \mathrm{mL})$ among the tested drugs against $M$. canis in our study. A previous study from Iran also demonstrated the high in vitro antifungal activity of terbinafine against $M$. canis (5). However, it is less useful in the treatment of tinea capitis due to M. canis. The MICs of terbinafine against $M$. canis could be higher than the maximum concentration reported in hair, accounting for treatment failure (15). The MICs of terbinafine in combination with allicin significantly declined compared with terbinafine alone, indicating that their combination might enhance the treatment of terbinafine in tinea capitis due to $M$. canis.

The actual antifungal mechanism of allicin is not yet fully understood. It can cause oxidation of glutathione that results in a shift of the cellular redox potential, inducing apoptosis of fungal cells (16). Another transcriptome study revealed that allicin impaired the expression of genes coding for enzymes of amino acid metabolism, iron uptake, the respiratory chain, thiamine metabolism and proteasomal protein degradation of fungal cells (17). The $\mathrm{MIC}_{50}$ and $\mathrm{MIC}_{90}$ of allicin were 32 and $128 \mu \mathrm{g} / \mathrm{mL}$ in this study. However, a previous study showed a $\mathrm{MIC}_{50}$ of $0.098 \mu \mathrm{g} / \mathrm{mL}$ and $\mathrm{MIC}_{90}$ of $0.195 \mu \mathrm{g} / \mathrm{mL}$, which were obviously lower (14). Yamada and Azuma found MICs of Trichophyton, Epidermophyton, and Microsporum were as low as $0.78-6.2 \mu \mathrm{g} / \mathrm{mL}$ (7). Another previous study reported higher MICs $(16-32 \mu \mathrm{g} / \mathrm{mL})$ of Trichophyton to allicin (18), which were consistent with our study. Similar discrepancy had also observed in C. albicans. A previous study showed that the MIC range of allicin to tested $C$. albicans strains was $0.025-12.5 \mu \mathrm{g} / \mathrm{mL}$ (19). However, another study showed a very higher MIC range $(64-512 \mu \mathrm{g} / \mathrm{mL})$ of allicin to $C$. albicans strains (8). The discrepancy may be due to the difference of drug resource, tested strains and antifungal susceptibility testing assay. Allicin alone does not have strong antifungal activity, while synergistic antifungal activity of allicin withitraconazole and terbinafine. Allicin may be an adjuvant therapy to traditional itraconazole and terbinafine therapy in tinea capitis caused by $M$. canis to reduce the course of treatment or drug dosage.

In conclusion, we described the antifungal susceptibility of M. canis to allicin, fluconazole, itraconazole, terbinafine and griseofulvin and investigated the combined antifungal activity of allicin with antifungal drugs. A major limitation of this work is that resistant strains were not tested. Our study revealed that the combination of allicin with itraconazole and terbinafine may represent an attractive perspective for the development of new management strategies for tinea capitis due to $M$. canis. Further in vivo studies are needed to validate our findings.

\section{DATA AVAILABILITY STATEMENT}

The datasets presented in this study can be found in online repositories. The names of the repository/repositories and accession number(s) can be found in the article/supplementary material.

\section{AUTHOR CONTRIBUTIONS}

YZ and YX contributed to conception and design of the study. YZ and JC recruited patients and collected data. YZ wrote the first draft of the manuscript. All authors contributed to the article and approved the submitted version. 


\section{REFERENCES}

1. Zhan P, Li D, Wang C, Sun J, Geng C, Xiong Z, et al. Epidemiological changes in tinea capitis over the sixty years of economic growth in China. Med Mycol. (2015) 53:691-8. doi: 10.1093/mmy/myv057

2. Khurana A, Sardana K, Chowdhary A. Antifungal resistance in dermatophytes: recent trends and therapeutic implications. Fungal Genet Biol. (2019) 132:103255. doi: 10.1016/j.fgb.2019.103255

3. Chadeganipour M, Nilipour S, Havaei A. In vitro evaluation of griseofulvin against clinical isolates of dermatophytes from Isfahan. Mycoses. (2004) 47:503-7. doi: 10.1111/j.1439-0507.2004.01050.x

4. Hsiao YH, Chen C, Han HS, Kano R. The first report of terbinafine resistance Microsporum canis from a cat. J Vet Med Sci. (2018) 80:898900. doi: 10.1292/jvms.17-0680

5. Abastabar M, Jedi A, Guillot J, Ilkit M, Eidi S, Hedayati MT, et al. In vitro activities of 15 antifungal drugs against a large collection of clinical isolates of Microsporum canis. Mycoses. (2019) 62:1069-78. doi: 10.1111/myc.12986

6. Liu S, Hou Y, Chen X, Gao Y, Li H, Sun S. Combination of fluconazole with non-antifungal agents: a promising approach to cope with resistant Candida albicans infections and insight into new antifungal agent discovery. Int $J$ Antimicrob Agents. (2014) 43:395-402. doi: 10.1016/j.ijantimicag.2013.12.009

7. Yamada Y, Azuma K. Evaluation of the in vitro antifungal activity of allicin. Antimicrob Agents Chemother. (1977) 11:743-9. doi: 10.1128/AAC.11.4.743

8. Guo $\mathrm{N}, \mathrm{Wu} \mathrm{X}, \mathrm{Yu} \mathrm{L}$, Liu J, Meng $\mathrm{R}$, Jin J, et al. In vitro and in vivo interactions between fluconazole and allicin against clinical isolates of fluconazole-resistant Candida albicans determined by alternative methods. FEMS Immunol Med Microbiol. (2010) 58:193-201. doi: 10.1111/j.1574-695X.2009.00620.x

9. Vitale RG, Afeltra J, Dannaoui E. Antifungal combinations. Methods Mol Med. (2005) 118:143-52. doi: 10.1385/1-59259-943-5:143

10. Clinical and Laboratory Standards Institute. Reference Method for Broth Dilution Antifungal Susceptibility Testing of Filamentous Fungi. 3rd ed. Wayne, PA: Clinical and Laboratory Standards Institute (2017).

11. Shin S, Lim S. Antifungal effects of herbal essential oils alone and in combination with ketoconazole against Trichophyton spp. J Appl Microbiol. (2004) 97:1289-96. doi: 10.1111/j.1365-2672.2004.02417.x

12. Gupta AK, Cooper EA. Update in antifungal therapy of dermatophytosis. Mycopathologia. (2008) 166:353-67. doi: 10.1007/s11046-008-9109-0

13. Finkelstein E, Amichai B, Grunwald MH. Griseofulvin and its uses. Int J Antimicrob Agents. (1996) 6:189-94. doi: 10.1016/0924-8579(95)00037-2
14. Aala F, Yusuf UK, Khodavandi A, Jamal F. In vitro antifungal activity of allicin alone and in combination with two medications against six dermatophytic fungi. Afr J Microbiol Res. (2010) 4:380-5. doi: 10.5897/AJMR.9000386

15. Arabatzis M, Kyprianou M, Velegraki A, Makri A, Voyatzi A. Microsporum canis antifungal susceptibilities: concerns regarding their clinical predictability. Int $J$ Antimicrob Agents. (2010) 36:385-6. doi: 10.1016/j.ijantimicag.2010.06.032

16. Gruhlke MC, Portz D, Stitz M, Anwar A, Schneider T, Jacob $\mathrm{C}$, et al. Allicin disrupts the cell's electrochemical potential and induces apoptosis in yeast. Free Radic Biol Med. (2010) 49:1916-24. doi: 10.1016/j.freeradbiomed.2010.09.019

17. Yu L, Guo N, Meng R, Liu B, Tang X, Jin J, et al. Allicin-induced global gene expression profile of Saccharomyces cerevisiae. Appl Microbiol Biotechnol. (2010) 88:219-29. doi: 10.1007/s00253-010-2709-x

18. Pyun MS, Shin S. Antifungal effects of the volatile oils from Allium plants against Trichophyton species and synergism of the oils with ketoconazole. Phytomedicine. (2006) 13:394-400. doi: 10.1016/j.phymed.2005.03.011

19. Khodavandi A, Alizadeh F, Harmal NS, Sidik SM, Othman F, Sekawi Z, et al. Comparison between efficacy of allicin and fluconazole against Candida albicans in vitro and in a systemic candidiasis mouse model. FEMS Microbiol Lett. (2011) 315:87-93. doi: 10.1111/j.1574-6968.2010.02170.x

Conflict of Interest: The authors declare that the research was conducted in the absence of any commercial or financial relationships that could be construed as a potential conflict of interest.

Publisher's Note: All claims expressed in this article are solely those of the authors and do not necessarily represent those of their affiliated organizations, or those of the publisher, the editors and the reviewers. Any product that may be evaluated in this article, or claim that may be made by its manufacturer, is not guaranteed or endorsed by the publisher.

Copyright (c) 2021 Zhou, Xiao, Chao and Ma. This is an open-access article distributed under the terms of the Creative Commons Attribution License (CC BY). The use, distribution or reproduction in other forums is permitted, provided the original author(s) and the copyright owner(s) are credited and that the original publication in this journal is cited, in accordance with accepted academic practice. No use, distribution or reproduction is permitted which does not comply with these terms. 